\title{
A Novel Solvent System to Prepare Pellets of Active Pharmaceutical Ingredients Having Tacky Wet-Mass Forming Propensity By Extrusion Spheronization Technique
}

\author{
Dinesh M Morkhade* \\ Department of Pharmaceutical Sciences, Rashtrasant Tukadoji Maharaj Nagpur University Campus, India
}

Received Date: June 04, 2018; Published Date: July 17, 2018

*Corresponding author: Dinesh M. MorkhadeDepartment of Pharmaceutical Sciences, Rashtrasant Tukadoji Maharaj Nagpur University Campus;Piramal Healthcare UK Ltd, Piramal Pharma Solutions, Whalton Road, Morpeth, Northumberland NE61 3YA, United Kingdom, Fax: +44 (0) 1670562400;E-mail: dmmorkhade@gmail.com

\begin{abstract}
Itopride hydrochloride $(\mathrm{IH})$ is a prokinetic agent that has been widely used for the treatment of dyspepsia and other gastrointestinal conditions. Because of its short biological half-life, it is suitable candidate for sustained drug delivery systems. However, IH has propensity to form a tacky/sticky wet-mass when comes in contact with water, especially in presence of hydroxypropyl methyl cellulose (Hypromellose). This makes it extremely difficult to process IH into sustained release granules or pellets using hypromellose as a matrix-forming material and aqueous-based solvent system. Therefore, aim of this study was to explore an optimum solvent system that can produce discrete and spherical SR pellets of IH by extrusion spheronization technique. Various conventional and non-conventional solvent systems were evaluated, and it was found that water: PEG 400 system has potential to produce a non-tacky wet mass of IH in presence of other pharmaceutical excipients. Therefore, IH and excipients were mixed and granulated using purified water: PEG 400 (85:15 ratio) in a high shear granulator. The wet mass was extruded using screw extruder equipped with $1.0 \mathrm{~mm}$ screen. The extrudes were spheronized at $700 \pm 100 \mathrm{rpm}$ for $10 \mathrm{~min}$ and dried in fluid bed drier at a target bed temperature of $42^{\circ} \mathrm{C}$. Pellets were characterized for morphology, particle size, friability, flow property and dissolution (pellets filled in capsule). Results revealed that the purified water: PEG 400 (85:15 ratio) system can produce a non-tacky wet-mass of IH and pharmaceutical excipients including hypromellose, which can be processed successfully into discrete and spherical pellets with sustained release profile and satisfactory pharmacotechnical properties.
\end{abstract}

Keywords: Itopride HCl; Pellets;Sticky;Tacky; Hypromellose; PEG

Abbreviations: HCl: Hydrochloride; PEG: Polyethylene Glycol; IH: Itopride Hydrochloride; SEM: Scanning Electro Microscopy

\section{Introduction}

Itopride hydrochloride ( $\mathrm{IH})$ is a prokinetic benzamide derivative that has been widely used for the treatment of functional dyspepsia and other gastrointestinal conditions [1]. It is a combined D2 receptor antagonist and acetylcholinesterase inhibitor. It is highly soluble in water and possess short biological half-life (about $6 \mathrm{hrs)} \mathrm{[2].} \mathrm{Due} \mathrm{to} \mathrm{its} \mathrm{short} \mathrm{biological} \mathrm{half-life,}$ IH is suitable candidate for designing sustained drug delivery systems.

Pellets are multi-unit particulate system (MUPS) that offer many advantages over the conventional monolithic systems. Pellets mitigate "all or none" phenomenon, reduce intra-subject variability and prevent dose dumping [3]. Pellets can be filled in sachets, capsules or compressed using auxiliary excipients to form tablets. Pellets distribute uniformly throughout the gastro- intestinal tract and improve absorption of active pharmaceutical ingredients (APIs) [3]. Pellets can be produced by two general techniques; layering and extrusion-spheronization [4]. In layering technique, neutral cores are coated with API and excipient followed by a functional coating [5]. The layering technique is preferred when the amount of API is low in formulation (low dose APIs). On the other hand, extrusionspheronization is a simple and cost-effective technique, and preferred particularly when the amount of API is high (usually $>10 \%$ ) in formulation [6]. In extrusion-spheronization, in order to produce satisfactory extrudes and spheronize them into discrete and spherical pellets, it is imperative to obtain a wet-mass of desired consistency. It has been well acknowledged that water, as a solvent, has potential to produce discrete and spherical pellets of a variety of pharmaceutical APIs. However, 
there are several APIs, which have propensity to form a tacky/ sticky mass when comes in contact with water, and therefore these are extremely difficult to process using water or aqueousbased solvent systems into pellets by extrusion spheronization technique. IH is one such example; it forms a tacky/sticky wet mass when comes in contact with water, and therefore IH pellets have been prepared mostly using organic solvents and drug layering technique in the past [7-9].

During our research, we noticed that Itopride $\mathrm{HCl}$, Atazanavir and two new chemical entities (NCEs; coded as NCE 1 and NCE 2) have propensity to form a tacky wet-mass when comes in contact with water. Such wet mass is extremely difficult to handle and process. To mitigate this, we incorporated detackifiers such as talc, and lubricants such as magnesium stearate (dispersed homogeneously in solvent) in different amounts in formulation. However, these were ineffective in reducing the tackiness of the wet mass. We also evaluated numerous solvents such as water, isopropyl alcohol, methylene chloride, acetone and combinations thereof, however, none of these could produce satisfactory wet mass that is suitable for further processing (extrusion and spheronization). It is noteworthy that the organic solvents, when used to produce wet-mass for extrusion, evaporated quickly rendering the wet-mass extremely dry and brittle. Therefore, we explored a range of non-conventional solvent systems prepared using combination of water with glycerol, polyethylene glycol (PEG 400), polysorbate 80, triethyl citrate, poloxamer and sodium lauryl sulphate. Amongst these, water: PEG 400 (85:15) solvent system could successfully produce non-tacky wet mass for all four APIs mentioned earlier in the text (though the liquid: solid ratio varied for these APIs). Consequently, the pellets of these APIs were prepared using water: PEG system by extrusion spheronization technique and evaluated for pharmacotechnical properties. This study deals with the preparation and evaluation of IH pellets only.

\section{Materials and Methods}

\section{Materials}

Itopride $\mathrm{HCl}$ was received as a gift sample from D. K. Pharmachem Pvt., Ltd., Mumbai, India. Microcrystalline cellulose (Avicel PH 101, FMC Biopolymer, Ireland), hydroxypropyl methylcellulose (HPMC K15M, Dow Chemical Company, Midland), colloidal silicon dioxide (Aerosil 200 pharma, Evonik Industries, Germany), talc (Imerys talc, Italy), polyethylene glycol (PEG 6000 and 400, BASF Corporation, Washington, US), glyceryl mnostearate (Univar Ireland Ltd. Ireland), polysorbate 80 (Brenntag Chemicals Distribution, Dublin, Ireland), magnesium stearate (Peter Greven, Germany) were used. The other chemicals were of analytical grade, purchased locally and used.

\section{Preparation of Itopride $\mathrm{HCl}$ pellets}

The composition of pellets is shown in Table 1. Itopride $\mathrm{HCl}$, microcrystalline cellulose, hypromellose and PEG 6000 were dry mixed in a high shear granulator for 10 minutes at a slow impeller speed. Separately, GMS was dispersed in purified water (preheated and maintained at $70^{\circ} \mathrm{C}$ ) using a laboratory homogenizer. The dispersion was allowed to cool to room temperature, and Tween 80 and PEG 400 were added to this. This was added to the dry mix of high shear granulator at a slow impeller speed. The resulting wet mass was mixed at fast impeller and fast chopper speed for $1 \mathrm{~min}$. The extrudes were prepared using Extruder 20 (Caleva Process Solutions Limited, England) equipped with 1.0 $\mathrm{mm}$ screen. These were then spheronized (using Spheronizer MBS, Caleva Process Solutions Limited, England) at $700 \pm 100$ rpms using a $3 \times 2 \mathrm{~mm}$ chequer plate for $10 \mathrm{~min}$. The pellets were dried in fluid bed dryer at $42 \pm 2^{\circ} \mathrm{C}$ for $3 \mathrm{hrs}$. and characterized for various physicochemical properties.

Table 1: Composition of Itopride $\mathrm{HCl}$ sustained release pellets.

\begin{tabular}{|c|c|c|c|}
\hline Ingredient & $\begin{array}{c}\text { Quantity per } \\
\text { capsule (mg) }\end{array}$ & $\mathbf{\%} \mathbf{w} / \mathbf{w}$ & $\begin{array}{c}\text { Quantity per } \\
\text { batch (g) }\end{array}$ \\
\hline Itopride HCl & 150 & 54.7 & 225 \\
\hline $\begin{array}{c}\text { Microcrystalline } \\
\text { cellulose (Avicel PH } \\
\text { 101) }\end{array}$ & 80 & 29.2 & 151.5 \\
\hline $\begin{array}{c}\text { Hydroxypropyl methyl } \\
\text { cellulose (HPMC } \\
\text { K15M) }\end{array}$ & 28 & 10.2 & 9 \\
\hline $\begin{array}{c}\text { Polyethylene glycol } \\
\text { (PEG 6000) }\end{array}$ & 5 & 1.8 & 9 \\
\hline $\begin{array}{c}\text { Glyceryl monstearate } \\
\text { Polysorbate 80 (Tween } \\
80 \text { ) }\end{array}$ & 1.8 & 0.7 & 0.3 \\
\hline $\begin{array}{c}\text { Water: PEG 400 } \\
\text { (85:15) }\end{array}$ & $9.0^{*}$ & 3.3 & $\begin{array}{c}22.6 \% \mathrm{w} / \mathrm{w} \\
\text { of dry mix } \\
\text { weight }\end{array}$ \\
\hline
\end{tabular}

\section{Evaluation of pellets}

\section{Friability}

A $10 \mathrm{~g}$ sample was sieved on ASTM 40 mesh in a sieve shaker (V81, Glen Creston Ltd., England) at $2 \mathrm{~mm}$ vibration-amplitude for $2 \mathrm{~min}$. Sieve shaker was used to standardize sieving step and thus to mitigate variations those otherwise would occur due to manual sieving and handling. Retained portion was retrieved carefully and charged to glass container $(105 \mathrm{~mL})$, which was then secured in granule friabilator (EGF-1, Electrolab, India). Sample was oscillated at 200 strokes per min for $3 \mathrm{~min}$ and sieved again through ASTM 40 mesh by sieve shaker before weighing. The method was optimized based on our experience to discriminate adequately between the various pellets formulations with this instrument.

\section{Particle size}

For particle size determination, about $1 \mathrm{~g}$ sample was placed on the conveyor belt of particle size analyzer (SystemPartikel-Technik, RODOS T4.1, Sympatec, GmbH), and particle size was determined at primary pressure of 3.1 bar and vacuum depression of $70 \pm 5$ mbar using R6 0.5/9.0, $1750 \mu \mathrm{m}$ lens. 


\section{Novel Approaches in Drug Designing \& Development}

\section{Ring shear testing}

The flow was also evaluated using ring shear tester (Dietmar Schulze ring shear tester, RST-XS, Germany). Pellets were filled gently in sample cell and the excess material was removed from surface, which was then smoothened using a scraper without applying any stress to the sample. The cell was placed on a mounting stand and the lid was placed on it. Loading and tie rods were attached to the lid. During the measurements of normal load, pre-shear was adjusted at $5000 \mathrm{~Pa}$. It is the load under which the sample is consolidated and kept in a steady state. Shearing was then proceeded at lower normal loads of 1000, 2000, 3000 and $4000 \mathrm{~Pa}$ consequently.The ratio of the consolidation stress to unconfined yield strength is termed as flow function index (ffc); higher ffc values indicate better flow. Results were analyzed using RST Control 95 software.

\section{Drug Release}

The dissolution study was carried out in $0.1 \mathrm{~N} \mathrm{HCl}$ for $2 \mathrm{~h}$ followed by phosphate buffer $\mathrm{pH} 6.8$ for $10 \mathrm{~h}$ using USP type II dissolution test apparatus at $50 \mathrm{rpm}$. For dissolution study, 274 mg pellets were filled in capsule and dissolution performed on 6 units. The percentage drug release was plotted against time to determine the release profile. The drug release kinetics determined using equations as described in literature [10].

\section{Results and Discussion}

IH has propensity to form a tacky wet-mass when comes in contact with water, specially in presence of some commonly used pharmaceutical excipients such as hydroxypropyl methyl cellulose. This makes it extremely difficult to process IH into sustained release granules or beads (using extrusionspheronization method) by aqueous granulation technique. Therefore, aim of this study was to explore suitable solvent system (aqueous based) to prepare sustained release IH pellets by extrusion spheronization technique using hypromellose as a matrix forming material.

To mitigate tackiness of the wet mass, we initially incorporated detackifier such as talc, and lubricant such as magnesium stearate (dispersed homogeneously in solvent) in different amounts in formulation. However, these excipients could not substantially reduce the tackiness of the wet mass. The organic solvents, when used alone, yielded extremely dry and brittle wet-mass that was not suitable for extrusion. Therefore, we explored a range of non-conventional solvent system prepared using combination of purified water with glycerol, polyethylene glycol (PEG 400), poloxamer and sodium lauryl sulphate in various different concentrations. Amongst these, water: PEG 400 in 85:15 system yielded wet mass that could be extruded and spheronized successfully to produce discrete and spherical sustained release pellets of IH.

The SEM (Figure 1) revealed that the purified water: PEG 400 solvent system could produce considerably discrete, uniform and round pellets of IH-hypromellose combination.The properties of sustained release IH pellets produced by extrusionspheronization technique are shown in Table 2.

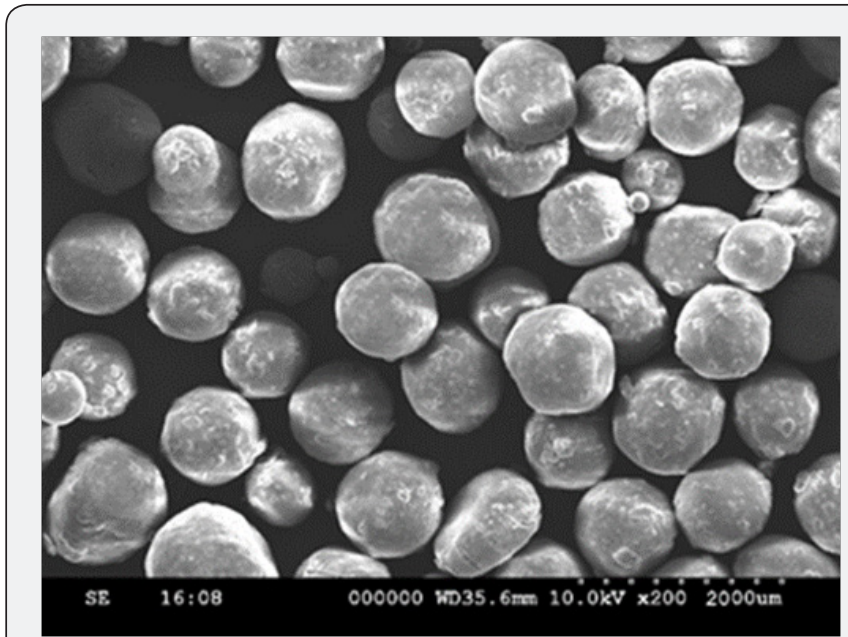

Figure 1: SEM of pellets produced using $\mathrm{IH}$, hypromellose and water: PEG 400 solvent system by extrusion spheronization technique.

Table 2: Properties of Itopride $\mathrm{HCl}$ pellets.

\begin{tabular}{|c|c|}
\hline Pellet characteristics & Value \\
\hline Bulk density $(\mathrm{g} / \mathrm{ml})$ & $0.87 \pm 0.006$ \\
\hline Taped density $(\mathrm{g} / \mathrm{ml})$ & $0.99 \pm 0.002$ \\
\hline Carr's index & 12 \\
\hline Hausner ratio & 1.14 \\
\hline FFC (Ring shear test) value & 14 \\
\hline Overall flow description & Good/ free flowing \\
\hline Mean Particle size (by sympatec) & $1.06 \mathrm{~mm}(0.72-1.31)$ \\
\hline Friability & $0.01 \%$ \\
\hline Drug release kinetics & $\begin{array}{c}\text { Zero order (Correlation co- } \\
\text { efficient 0.9884) }\end{array}$ \\
\hline
\end{tabular}

The mean particle size of pellets was $1.06 \mathrm{~mm}$, which was in accordance with the extrusion screen used $(1.0 \mathrm{~mm})$. The Carr's index and Hausner ratio indicated good flow property, whereas the ring shear testing (ffc values) suggested free flowing characteristics of the pellets. The friability of IH pellets was insignificant, which indicates their potential to withstand handling, transport and further processing (such as capsule filling).

The drug release profile of IH pellets is shown in Figure 2. It depicts that the formulation (composition) used for this study could effectively sustain drug release for 12 hours. The drug release was faster for initial $2 \mathrm{hrs}$ followed by a gradual release thereafter up to $10 \mathrm{hrs}$. The initial fast release might be attributed to the time taken by hypromellose matrix to swell and form release retarding barriers/domains in pellets. Because of the matrix system, it was anticipated that the drug release from IH pellets will follow Higuchi square root kinetics, however, the pellets exhibited zero-order drug release profile (Table 2). This might be due to the presence of PEG 400 and 
PEG 6000 in formulation, which are known to facilitate drug solubilization, diffusion (through the matrix) and subsequently the drug release from formulation. It appears that a combination of higher amount of PEGs and hypromellose as a matrix agent might have resulted in a zero order drug release from IH pellets.

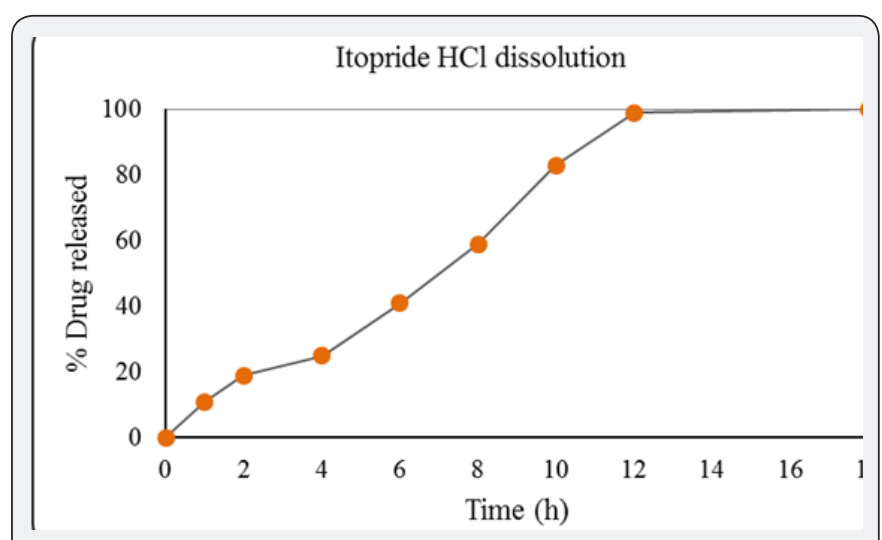

Figure 2: Drug release profile of Itopride $\mathrm{HCl}$ pellets.

\section{Conclusion}

Itopride $\mathrm{HCl}$ has propensity to form a tacky wet-mass when comes in contact with water; this effect is more prominent in presence of some commonly used pharmaceutical excipients such as hydroxypropyl methyl cellulose (hypromellose). Therefore, it is challenging to produce sustained release IH pellets using hypromellose, aqueous based solvent system and extrusion-spheronization technique.In view of this, present study was undertaken with an objective to derive an optimum solvent system (aqueous based) that can produce discrete and spherical pellets of IH using hypromellose as a matrix material and extrusion-spheronization as a pelletization technique. For this purpose, detackifier, lubricant, a range of individual solvents and combinations thereof were investigated, and it was found that the purified water: PEG 400 (85:15) solvent system has potential to produce a wet-mass that can be extruded and spheronized successfully into discrete and spherical pellets of IH. Results revealed that purified water: PEG 400 is a promising solvent system that can be explored to granulate APIs having a tacky wet mass forming propensity to process them into sustained release granules, pellets or other suitable dosage forms.

\section{References}

1. Stevens JE, Russo A, Maddox AF, Rayner CK, Phillips L, et al. (2008) Effect of itopride on gastric emptying in longstanding diabetes mellitus. Neurogastroenterol Motil 20(5): 456-463

2. Summary of product characteristics for PROGIT $50 \mathrm{mg}$ film coated tablets.

3. Dey NS, Majumdar S, Rao MEB (2008) Multiparticulate Drug Delivery Systems for Controlled Release. Tropical Journal of Pharmaceutical Research 7(3): 1067-1075.

4. Supriya P, Rajni B, Rana AC (2012) Pelletization techniques: A literature review. Int Research Journal of Pharmacy 3(3):43-47

5. Ahir AA, Mali SS, Hajare AA, Bhagwat DA, Patrekar PV (2015) Pelletization Technology: Methods and Applications -A Review. Res J Pharm Tech 8 (2): 131-138

6. Muley S, Nandgude T, Poddar S (2016) Extrusion-spheronization a promising pelletization technique: In-depth review. Asian j pharm Sci 11(6): 684-699

7. Soni S, Sharma D, Ola S, Naruka PS (2013) Formulation and evaluation of Itopride $\mathrm{HCl}$ sustained release pellets. Innovare J Life Sci 1(2): 11-16

8. Rao PSS, Babu GR, Praveen TK, Surekha PSL, Shekhar MC (2014) Formulation and evaluation of Itopride $\mathrm{HCl}$ sustained release pellets. J Pharm Sci Res 5(5): 2074- 2083

9. Biswal B, Karna N (2014) A novel approaches for formulation of Itopride HCl MUPS sustained release tablets. Int J Pharm 4(1): 194-203

10. Costa P, Lobo JMS (2001) Modeling and comparison of dissolution profiles. Eur J Pharm Sci 13(2): 123-133.

\section{Your next submission with Juniper Publishers} will reach you the below assets

- Quality Editorial service

- Swift Peer Review

- Reprints availability

- E-prints Service

- Manuscript Podcast for convenient understanding

- Global attainment for your research

- Manuscript accessibility in different formats

( Pdf, E-pub, Full Text, Audio)

- Unceasing customer service

Track the below URL for one-step submission https://juniperpublishers.com/online-submission.php 